\title{
Sobre la autonomía heterodoxa y las debilidades del concepto ${ }^{1}$
}

\section{About the heterodox autonomy and the concept's weakenesses}

\author{
MARIA CECILIA MIGUEZ \\ Investigadora Adjunta del Consejo Nacional de Investigaciones \\ Científicas y Técnicas (IDEHESI). Correo electrónico: \\ mmccmiguez@gmail.com
}

\section{Resumen}

La doctrina de la "autonomía" en América Latina, y la versión argentina elaborada por Juan Carlos Puig, constituye un aporte ineludible para el área de las Relaciones Internacionales. Este artículo, aspira a reflexionar sobre algunas las limitaciones del concepto de autonomía heterodoxa para divisar la dificultad que suscita la aplicación de conceptos a los casos históricos, sin aplicar un enfoque de historia total. En este sentido, consideramos necesario incorporar al análisis, un enfoque histórico que permita balancear las necesidades de encontrar patrones y categorías con la importancia de comprender la complejidad tanto de la política como del escenario internacional.

\section{Palabras clave}

América Latina - Argentina Autonomía Heterodoxa - Juan Carlos Puig

\section{Abstract}

The doctrine of "autonomy" in Latin America, and the Argentine version elaborated by Juan Carlos Puig, is in an unavoidable place for the area of international relations. This article is a summary of some of the limitations of the concept of heterodox autonomy for the difficulty of the task of applying historical concepts. In this sense, we consider it necessary to include analysis, a historical approach that allows us to balance the needs of finding patterns and categories with the importance of understanding the complexity of both politics and the international scenario.

\section{Keywords}

Latin America - Argentina Heterodox Autonomy - Juan Carlos Puig

\footnotetext{
${ }^{1}$ Este trabajo constituye una versión reducida de otro de más largo alcance publicado en Míguez, María Cecilia (2017) La autonomía heterodoxa y la clasificación de las políticas exteriores en la Argentina, Revista de Relaciones Internacionales, Estrategia y Seguridad de la Universidad Militar de Nueva Granada, Colombia, 12(2):207-229.
} 
Perspectivas Revista de Ciencias Sociales - ISSN 2525-1112|Año 3 No. 6 Julio-Diciembre 2018, pp. 209-220

\section{Introducción}

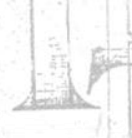

La doctrina de la "autonomía" en América Latina, y su versión en la Argentina elaborada por Juan Carlos Puig a lo largo de varios trabajos, constituye a juicio de esta autora, una elaboración ineludible tanto para los teóricos y especialistas en el área de las Relaciones Internacionales, como para los dirigentes políticos. Sin duda fue reflejo de las intenciones de construir proyectos alternativos a la dominación de los Estados Unidos en la región, en pleno período de la Guerra Fría. Es por ello el más importante punto de partida para quienes creemos que es posible construir una inserción internacional democrática y soberana, que construya espacios de poder para la afirmación y la defensa de los intereses de los países dependientes del sistema internacional que, por definición, son contradictorios con los de aquéllos que se benefician con esas relaciones asimétricas. Es en este sentido que me permito reflexionar sobre algunas de las limitaciones del concepto de autonomía heterodoxa, con la finalidad de resaltar la importancia de la concurrencia de distintas potencias que disputan predominio mundial -más aún en el recrudecido contexto actual- y evitar caer en la fantasía de que recostarse sobre el poder de otros países predominantes es por sí solo garantía de autonomía.

Uno de los supuestos de la teoría de Puig es la posibilidad de que en el contexto de relaciones internacionales asimétricas se puedan llevar a cabo estrategias autonomizantes enmarcadas en dos escenarios tipo: la dependencia nacional y la autonomía heterodoxa. "En el primer caso, las elites nacionales aceptan las consecuencias de las relaciones asimétricas, pero tratan de sacar el mayor provecho posible de tales relaciones aun a riesgo de conflictos importante debido a la 'miopía' de la potencia dominante; en el segundo, reivindican aspectos cada vez más significativos para su decisión propia" (1988: p.20).

Este autor, quien se desempeñó como canciller durante la presidencia de Héctor Cámpora, consideró como ejemplo de una política de autonomía heterodoxa a la "tercera posición", formulación coherente de política exterior de los primeros gobiernos peronistas, en la que había coincidido el margen potencial de decisión autónoma del país con el margen actual (1988: p.33). La categoría teórica es definida como un estadio donde lo que Puig llama "supremos repartidores nacionales" discrepan con la potencia dominantes en tres cuestiones: 1) en el modelo de desarrollo interno que puede no coincidir con las expectativas de la metrópoli; 2) en las vinculaciones internacionales que no sean globalmente estratégicas $y, 3$ ) en el deslinde entre el interés nacional de la potencia dominante y el interés estratégico del bloque (1984: p. 78).

Históricamente, lo aplica -si bien distinguiendo matices- para otros casos en el período 1955-1973. Se refiere así a la política exterior aplicada por los gobiernos de Frondizi, Illia, Levingston y Lanusse (Puig, 1980: p.157). Los gobiernos de Cámpora y Perón habrían profundizado la línea de este último, de nuevo en el marco de la "tercera posición", y de acuerdo con su planteo, la última dictadura militar fue un período de "persistencias autonomistas heterodoxas en un contexto político disfuncional y económico dependentista" (Puig, 1988: p.38). 
Tenemos aquí políticas exteriores que, si bien pueden tener rasgos en común, también tienen importantes diferencias. En cuanto a lo que podría asemejarlos, es en cierta medida la relación con los Estados Unidos ya que en todos los casos pueden citarse elementos donde existe un relativo margen de autonomía.

Uno de los aspectos más endebles es que la autonomía es medida en forma exclusiva respecto del país que se considera hegemónico en el período, pero se desconoce la disputa por esferas de influencia por parte de las distintas potencias. Durante la etapa posterior a 1955, el margen de autonomía es analizado solamente respecto de la relación con los Estados Unidos. Esa falencia desemboca en la afirmación de que la política exterior de la última dictadura militar puede ser entendida como de autonomía heterodoxa, al igual que el caso de la "tercera posición" de los primeros gobiernos peronistas. Puig intenta resolver esa contradicción sosteniendo que la orientación autonomista del gobierno militar "no llegó nunca a estructurarse en un proyecto coherente y global" y que

(...) no se podía mantener una política exterior autonomista sin participación del pueblo y de las organizaciones populares en su formulación e implementación, así como tampoco era posible sustentarla en un esquema económico que incrementaba la dependencia y, sobre todo, la vulnerabilidad del país desde el punto de vista de su seguridad (Puig, 1988: p.39).

Los autores Mario Rapoport y Claudio Spiguel (2005) fueron los primeros en llamar la atención sobre estas debilidades, y sostuvieron acertadamente que el esquema interpretativo "opaca la concurrencia entre distintas potencias en el mercado mundial contemporáneo y en el sistema internacional de relaciones, tanto como la particular incidencia de estas en las clases dirigentes argentinas a lo largo del siglo XX" (p.50).

Si analizamos la historia de las relaciones internacionales en Argentina, uno de los aspectos que podemos detectar a partir de los estudios realizados desde la década de 1980 en adelante, en especial los de Mario Rapoport (1981) y Carlos Escudé (1983), es la concurrencia de varias potencias disputando el predominio económico y político en el país. Especialmente los trabajos sobre la relación triangular de la década de 1920 elaborados por Mario Rapoport dieron cuenta de este aspecto estructural de la inserción internacional argentina. Por ello, centrar el análisis de la política exterior desde la posguerra en adelante, únicamente, en la relación con los Estados Unidos, presenta problemas rápidamente. Puig comete ese error porque soslaya la concurrencia a la que nos referimos y, por lo tanto, descuida el análisis sobre las condiciones que, en algunos casos, posibilitan grados de acercamiento o alejamiento de la potencia hegemónica principal. Algo que caracterizó sin duda al contexto internacional de la guerra fría. Este aspecto conduce a un error prescriptivo: si el problema es el acercamiento unilateral a la potencia hegemónica, cualquier tipo de diversificación de las relaciones exteriores es entendido como rasgo de autonomía. Así, la estrategia multilateral aparece como 
sinónimo de independencia. Se descuida, en efecto, otro aspecto, que es la existencia de otras relaciones asimétricas, las cuales también significan nuevos lazos de dependencia, con otras potencias secundarias.

Por lo tanto, intentaremos a partir del desarrollo breve de los casos de la política exterior de Arturo Frondizi, Artuto Illia y la última dictadura militar (19761983) -para seleccionar algunos ejemplos- afinar la clasificación tradicional de Puig que coloca a las tres primeras dentro de la autonomía heterodoxa, y recuperar la importancia de la concurrencia de varias potencias para explicar y distinguir los comportamientos de los gobiernos en el sistema internacional. No toda política exterior con margen de autonomía respecto de una o varias potencias implica necesariamente la promoción de una inserción internacional autónoma. En primer lugar, porque dicho margen se define respecto de algo, de algún país y no necesariamente de todos. En segundo, porque esa tendencia puede expresarse en algunas de las áreas de la política y no en todas.

\section{La autonomía heterodoxa del proyecto desarrollista}

Respecto de la política exterior de Frondizi, existen trabajos que la identificaron como "independiente", como el caso de Archibaldo Lanús (1986: 74), mientras que otros focalizaron en los aspectos de apertura respecto del capital extranjero y afirmaron lo contrario. También fue calificada como una estrategia de "cornisa", ya que pretendía caminar por un pequeño andarivel, que le otorgara una relativa "independencia" frente a los Estados Unidos (Rapoport, 1997: p.353). Comprender a fondo dicha política internacional requiere partir de un complejo análisis de variables internas y externas. Alain Rouquié (1967) interpreta la afirmación del poder personal de Frondizi y los rasgos "independientes" de su política, partiendo de la necesidad del gobierno de mantener y resguardar un ámbito que le otorgara prestigio y cierta popularidad, para contrarrestar la gran crisis y pérdida de apoyo en la política interna, es decir, "proveer consensos que compensaran la impopularidad de las medidas adoptadas en el terreno económico y social" (p.146-147). Una posición similar sostiene Robert Potash (1981) cuando afirma que el ámbito de las relaciones exteriores, en especial después de 1961, ofrecía a Frondizi la oportunidad de recuperar su imagen, para demostrarles a los radicales intransigentes y a los nacionalistas que seguía sosteniendo sus principios e intenciones de desempeñar un papel independiente en las políticas hemisféricas e inclusive aspiraría a un reconocimiento en el concierto mundial (p.450).

Los márgenes de autonomía existentes en la política exterior fueron especialmente en el ámbito político y diplomático y redundaron en altos costos para su gobierno, ya que cuentan entre las causas del golpe militar que lo derrocó en 1962. Pero, por otro lado, sería erróneo afirmar que eran el correlato de un proyecto vinculado con el nacionalismo económico, dadas las características del modelo desarrollista y su estrecha vinculación con el capital extranjero. Es decir que en los aspectos económicos, hubo acercamiento con los Estados Unidos y con la Unión Soviética, las dos superpotencias del período (Míguez, 2011). Así lo expresaba también un informe de coyuntura del Departamento de Estado que consideraba que 
Frondizi parecía dispuesto a seguir la tendencia marcada por el régimen de Aramburu hacia una cada vez mayor cooperación internacional y que había solicitado estrechar la relación con los Estados Unidos. ${ }^{2}$

Entre los elementos que explican las posibilidades de aplicar una política con margen de autonomía respecto de los Estados Unidos se destaca la propia dinámica de la guerra fría en la región, y el espacio que generaba la competencia entre las superpotencias. Las Fuerzas Armadas y el más duro antiperonismo utilizaron el argumento de la cercanía de Frondizi con las ideologías de izquierda para erosionar un poder ya debilitado a partir de la ruptura de la alianza con el peronismo. En la cancillería, los funcionarios conservadores, liberales y los más variados promotores de los vínculos con la potencia del norte reaccionaron contra la orientación internacional del presidente y de su colaborador cercano, Rogelio Frigerio.

\section{El caso del nacionalismo de gobierno del radicalismo del pueblo}

En el caso del gobierno de Arturo Illia (1963-1966), el distanciamiento respecto de los Estados Unidos incluyó, a diferencia del caso anterior, cuestiones centrales de la economía. El gobierno de Arturo Illia contó con un poder restringido desde el inicio, ya que asumió sin haber logrado la mayoría en la Cámara de Diputados y habiendo sido electo con menos del $25 \%$ de los votos, mientras que los votos en blanco alcanzaron aproximadamente el 20. Ello, junto con el complejo escenario de proscripción del peronismo y de enfrentamientos entre fracciones de las Fuerzas Armadas atentaría contra la legitimidad de su mandato.

La política exterior llevada adelante mantuvo en algunos aspectos de la agenda, márgenes de autonomía respecto de los Estados Unidos y de las potencias en general. Ello se expresó en medidas de orden económico y otras de orden políticodiplomático y estratégico. En cuanto a las primeras, desde su campaña electoral, el presidente hizo foco en la necesidad de anular los contratos petroleros ratificados durante la presidencia de Arturo Frondizi, e iniciar una investigación al respecto, situación que generó preocupación en Estados Unidos, principal país de origen de las empresas inversoras del rubro. La anulación fue una de sus primeras medidas de gestión a pesar de las presiones de Averell Harriman -enviado del presidente John Kennedy que visitó Buenos Aires entre el 8 y el 10 de noviembre de 1963-y del senado de los Estados Unidos (Autor/a, 2016). Asimismo, también diferenciándose de los desarrollistas, Illia se negó a renovar el Acuerdo de Garantía de Inversiones firmado por su antecesor, y a lo largo de su mandato, se aplicó una política heterodoxa, que incluyó no solamente una suba de salarios a partir del establecimiento en 1964 de la Ley de Salario Mínimo Vital y Móvil, sino que aplicó controles de precios que alcanzaron a productos de la canasta familiar, bienes de

\footnotetext{
2 "The Outlook for Argentina. National Intelligence Estimate" (5 de agosto de 1958), Foreign Relation of the United States 1958-1960, vol. V, p. 495. En una nota al pie, se aclara especialmente: "durante el régimen de Aramburu, Argentina acompañó al Banco Mundial y al FMI ratificando la Carta de la Organización de los Estados Americanos, siendo el último país en hacerlo, y aprobó la resolución anticomunista firmada en Caracas en 1954".
} 
consumo especiales y tarifas públicas (Autorla, 2014). Se regularon las remesas de utilidades, el pago de intereses y dividendos, la industria automotriz y la farmacéutica. En este último caso se implementó una Ley de Medicamientos que regulaba tanto los procedimientos para aprobar nuevas especialidades medicinales y los requisitos a cumplir, como los márgenes razonables de ganancia en la cadena de producción y comercialización y que obligó al gobierno a enfrentar a un importante lobby de los laboratorios transnacionales.

Este tipo de políticas iban a contramano de los planes de ajuste promovidos por el FMI en ese momento, lo que complicaba la relación con el organismo y, por lo tanto, las líneas de crédito internacional. En 1965 se producía un importante vencimiento de los acuerdos firmados previamente con el fondo, y para negociar un crédito stand by con dicho organismo, debía aceptarse un importante cambio en la regulación del movimiento de capitales, una devaluación del peso y una política monetaria restrictiva. Ello constituyó un punto clave de tensión externa e interna (Autor/a, 2016).

En cuanto a las cuestiones político diplomáticas y estratégicas, en el contexto de la guerra fría, el gobierno radical llevó a cabo una política exterior guiada por el "idealismo krausista" y el "universalismo", ambos de inspiración yrigoyenista (Simonoff, 2007); e hizo pie en la necesidad del desarrollo nacional, la integración continental y la confianza en la Alianza para el Progreso.

En el caso de este gobierno podemos afirmar que existió una política de relativa autonomía respecto del modelo de desarrollo impulsado por la potencia dominante en la región, coincidiendo con una de las características centrales del concepto de autonomía heterodoxa, y diferenciándose claramente de la conducta del gobierno de Frondizi. En términos diplomáticos y militares, la cuestión es más compleja de definir, en tanto coexistieron políticas que confrontaron con los Estados Unidos, como la negativa al envío de tropas a Santo Domingo, con otras donde el canciller Zavala Ortiz intentaba mostrar acercamiento. Ejemplo de esto último es la firma conjunta en el mes de mayo de 1964, entre el canciller y el embajador de los Estados Unidos Mc Clintock, de un convenio de cooperación militar que se basaba en la aceptación del nuevo concepto de la seguridad continental, reemplazando la doctrina de la amenaza externa por la interna, es decir, la ideológica. Era la base de la nueva Doctrina de la Seguridad Nacional ${ }^{3}$. Específicamente se trataba de un Memorándum de entendimiento respecto de un Programa de Asistencia Militar (Military Assistance Program MAP), que refrendaba la cooperación en el marco de la amenaza castrista-comunista al interior de cada uno de los países latinoamericanos. Implicaba realmente un hito importante en la estrategia de seguridad estadounidense en la región (Autor/a, 2015).

En síntesis, de los casos analizados, el del gobierno de Illia, con su complejidad, es el que más se ajusta a la definición de autonomía heterodoxa elaborada por Puig.

\footnotetext{
${ }^{3}$ Para un análisis del devenir del concepto de seguridad internacional ver Cujabante, 2009.
} 
Profundización de la dependencia durante la última dictadura militar: de autonomía nada...

El caso de la última dictadura militar es significativo, porque las transformaciones estructurales que impuso se tradujeron en una reprimarización de la estructura productiva, basada en una nueva relación de subordinación respecto de los Estados Unidos y de la Unión Soviética en términos de una nueva relación triangular (Rapoport y Spiguel, 2005: 55). Las medidas adoptadas tuvieron como consecuencia una apertura de la economía que provocó la quiebra de ramas enteras de la industria nacional, reorientando la economía a la exportación de unos pocos productos primarios y a la especulación, socavando las bases de cualquier tipo de inserción internacional autónoma.

En cuanto a los Estados Unidos y tal como lo demuestran varios autores se han especializado en el período, a pesar del carácter complejo de la relación entre Argentina y Estados Unidos, el apoyo económico a la dictadura por parte del Tesoro de los Estados Unidos y la banca privada liderada por David Rockefeller fue fundamental, no sólo inicialmente durante el gobierno de Gerald Ford sino también en el de James Carter, con quien habría mayores discrepancias políticas (Morgenfeld, 2013).

Al mismo tiempo, el gobierno fue mostrando rasgos de acercamiento económico cada vez más estrechos a intereses vinculados con la URSS. De acuerdo con Rapoport, en ello pesaban razones históricas -lazos existentes desde el primer peronismo y cultivados en especial desde 1971-, políticas -entendidas muchas veces como contrapeso de la injerencia de potencias occidentales tradicionales- y económicas -la colocación de productos agroexportables (Rapoport, 2006: p. 640). En efecto, el gobierno militar ratificó una gran cantidad de acuerdos bilaterales negociados por José Ber Gelbard, ministro del tercer gobierno peronista, el comercio con la URSS se duplicó entre 1976 y 1979, y surgieron grandes emprendimientos de inversión y promoción industrial (Vacs, 1984). Se firmó un convenio pesquero para la pesca e industrialización del krill en el Atlántico Sur, se acordó la concreción de proyectos de centrales hidro y termoeléctricas, insumos para la central nuclear Atucha I, maquinarias y vehículos (Rapoport, 2006: p. 641).

A partir de la asunción de Carter en 1977, entramos en una etapa de relativo enfriamiento de la relación política entre Argentina y Estados Unidos (Vazquez Ocampo, 1987: p.119). El vínculo estuvo signado por una serie de tensiones en lo referido a la violación sistemática de los derechos humanos (aplicación del terrorismo de Estado), la transferencia de armamentos y la política nuclear del gobierno militar. A ello se agrega la permanencia de la Argentina en el Movimiento de No Alineados. Ante la feroz y sangrienta persecución llevada adelante por el Estado de los opositores políticos y sindicales con el argumento de la lucha antisubversiva, Estados Unidos incluyó a la Argentina en una política que buscaba denunciar los abusos contra los derechos humanos cometidos en el bloque soviético (Rapoport, 2006: p.629). Cabe destacar que fueron los complejos vínculos del país y la desconfianza generada por su inserción triangular, lo que explicaría la insistencia en la denuncia por parte de Estados Unidos, a diferencia de otras 
dictaduras latinoamericanas que también cometían ese tipo de violaciones promovidas por la Doctrina de la Seguridad Nacional y las estrategias militares de contrainsurgencia, como el caso de Chile (Rapoport, 2006: p.629). Los beneficios de la relación económica con la URSS se expresaron políticamente en organismos internacionales, donde los soviéticos se negaron sistemáticamente a que se cuestionara a la Argentina, frenando las críticas que surgían en foros internacionales por parte de países de Europa Occidental y de organizaciones independientes (Paradiso, 1993: p.176).

Por lo tanto, ello derivó en una disminución de la ayuda militar, que bajó de 32 millones de dólares a 15,7 millones, al mismo tiempo que se iniciaba otra controversia referida al desarrollo nuclear argentino, ya que el general dictador Jorge Rafael Videla se negó a ratificar el Tratado para la Proscripción de Armas Nucleares en América Latina, suscripto en 1967. Cabe destacar que incluso la dictadura militar liderada por Juan Carlos Onganía (1966 y 1970), de gran afinidad política, ideológica y económica con los Estados Unidos, tampoco lo había ratificado, con lo cual podría considerarse una postura tradicional del país.

Por último, en respuesta a su dependencia del mercado soviético y en función de los vínculos cultivados desde 1971 en adelante (Rapoport, 1988: p.182), Argentina rechazó el embargo cerealero de los Estados Unidos contra la Unión Soviética en respuesta a la invasión a Afganistán en 1980 (Lanús, 1984: p.116). Ello generó tensiones, pero detrás de algo que puede ser interpretado como "autonomismo" subyacía la dependencia argentina de las exportaciones de granos hacia la URSS que se había convertido en su principal importador (Rapoport, 2006: p. 631)

Sin embargo, las relaciones económicas basadas en el intercambio comercial pero fundamentalmente en el enorme endeudamiento proveniente de bancos occidentales, continuaron. La política de apertura financiera instalada a partir de 1977 fue en consonancia con las propuestas de la Comisión Trilateral, y generó un proceso de especulación y endeudamiento público y privado del que importantes bancos occidentales obtuvieron grandes beneficios. El paquete de medidas adoptadas por el ministro José Alfredo Martínez de Hoz tuvieron como consecuencia una retrogresión en la inserción de la economía en el mercado mundial, profundizando su carácter dependiente de las exportaciones agropecuarias (destrucción de gran parte del aparto industrial orientado a mercado interno) y del capital financiero internacional (Rapoport y Spiguel, 2005: p.540). Hay que destacar que la nueva legislación sobre radicación de capitales e inversión extranjera (Ley $N^{\circ}$ 21.382) posibilitó una abrumadura afluencia de capitales, y que para el período 1977-1980, Estados Unidos lideraba la inversión extranjera directa en el país con el 36,92\% de participación (Cisneros y Escudé, 1999, Parte III, Cap. IV, Las inversiones extranjeras)

Por lo tanto, a pesar del resentimiento inicial de algunos sectores y la presión de ciertos legisladores norteamericanos para que se aplicaran sanciones a la Argentina por violaciones a los derechos humanos, en octubre de 1978 se acordó incrementar las relaciones económicas (Cisneros y Escudé, 1999, Parte III, Cap. IV, 
Las relaciones comerciales). Una reunión entre Videla y el vicepresidente Walter Mondale en Roma posibilitó una autorización del Departamento de Estado a un importante crédito del Eximbank para garantizar el financiamiento de la represa hidroeléctrica Yaciretá (Rapoport, 2006: p.630). Una vez más, en la relación entre ambos países influían corrientes diversas y contradictoras de un lado y de otro.

Estos elementos nos impiden afirmar que esa política en efecto heterodoxa pudiera considerarse autónoma, sino que manejó un margen de distancia de las posiciones de la potencia del Norte en función de la propia dinámica de la guerra fría y su inserción triangular, y de cuestiones de política interna. Asimismo, con la asunción de Ronald Reagan se profundizaron lazos políticos que redundaron en la participación de militares argentinos en operaciones de apoyo a los contrarrevolucionarios nicaragüenses (Russell, 1988: p.117), y más adelante, hacia 1982, en el levantamiento de las sanciones económicas y militares previas (Aguirre Lanari, 1996: p.332).

Teniendo en cuenta todas estas cuestiones resulta contradictorio considerar a la última dictadura militar como una política de autonomía heterodoxa al igual que las dos descriptas anteriormente, especialmente porque se trató de un proyecto económico que profundizó los lazos de dependencia, aunque incorporara otros vínculos del sistema internacional a ese patrón periférico. Más allá de las aclaraciones que hace Puig, este tipo de estrategia se adecúa mejor a una dependencia heterodoxa, que a una calificación de autonomía.

\section{Conclusiones}

Partiendo de resaltar la importancia de retomar los enfoques autonomistas para pensar la política internacional argentina, este breve repaso ha pretendido hacer un comentario humilde sobre la dificultad que suscita la aplicación de conceptos a los casos históricos, sin aplicar un enfoque de historia total. Es decir que consideramos necesario incorporar al análisis conceptual y comparativo de las políticas internacionales del país, un enfoque histórico que permita balancear las necesidades de encontrar patrones y categorías con la importancia de comprender la complejidad tanto de la política como del escenario internacional. Por esa razón, consideramos que en cada caso de estudio se debe tener en cuenta, para enriquecer el análisis que utilice un enfoque autonomista:

1) cuáles son las potencias predominantes en el sistema mundial en ese momento, su relación y su vinculación con el país en cuestión;

2) interpretar el tipo de inserción económica internacional del país fomentada por la política pública, analizando condicionantes estructurales y medidas políticas, económicas y estratégicas implementadas en la coyuntura;

3) vincular las pujas en el Estado con las relaciones de fuerza en el plano de la política y la economía domésticas, e incluir dentro del bloque en el poder la relación de dependencia como factor interno;

4) evaluar la forma de Estado, teniendo en cuenta los niveles de autonomía relativa y el comportamiento de la dirigencia política, así como su 
posicionamiento político y diplomático respecto de cuestiones de agenda local e internacional;

5) analizar los vínculos con países a los que podríamos considerar "pares" en términos del tipo de la posición ocupada en el sistema internacional. Es decir, con aquellos que posibilitan las condiciones en las que es posible desarrollar una "autonomía horizontal" respecto de la contradicción principal entre potencias hegemónicas y países subordinados (Míguez, 2017 b) y;

6) ponderar el grado de afinidad, complementariedad, subordinación, oposición o confrontación del proyecto económico y político interno llevado adelante por el país y el de las potencias predominantes en el sistema internacional.

Para aportar a la discusión conceptual luego de haber analizado los citados casos históricos, dentro de todos los ejemplos que Puig señala como de "autonomía heterodoxa" podríamos distinguir:

a) períodos donde la política pública se orienta a la búsqueda de una inserción internacional autónoma, poniendo reparos a la injerencia de las potencias hegemónicas -una o varias- tanto en la política interna como en la política exterior, como los casos de la Tercera Posición de Juan Domingo Perón y en cierta medida el de Arturo Illia. En este sentido, habría un importante grado de confrontación con la potencia predominante, los Estados Unidos, en términos políticos, estratégicos y económicos (sin descuidar aspectos pragmáticos que el propio Puig considera necesarios). Y podríamos clasificarlos como "períodos de construcción de autonomía";

b) otros donde las políticas aplicadas profundizan una estructura de inserción internacional dependiente, es decir, asumiendo un importante nivel de afinidad o complementariedad subordinada entre el proyecto económico impulsado por el Estado y los requerimientos de algunas de las potencias del sistema internacional, pero se confronta en otros aspectos con los Estados Unidos sobre la base del redireccionamiento o diversificación de las relaciones exteriores, como el caso de la última dictadura militar en la Argentina. Rapoport y Spiguel lo han considerado como "diversificación de la dependencia" (Rapoport y Spiguel, 2005: p.50);

c) otros donde coexiste una afinidad en términos de la política económica interna y externa, entre el país y la potencia hegemónica del período, con la búsqueda de márgenes de autonomía en las dimensiones políticas, diplomáticas y estratégicas. Ese sería el caso del gobierno de Arturo Frondizi y en cierto modo de Raúl Ricardo Alfonsín (Míguez, 2013: p. 280). Aquí, el análisis debería orientarse a comprender qué elementos son los que explican esa coexistencia, teniendo especialmente en cuenta el accionar de otros actores políticos del sistema internacional, así como las contradicciones políticas en el plano interno. Si el grado de afinidad en términos de política económica con las potencias hegemónicas en el sistema internacional es 
Perspectivas Revista de Ciencias Sociales - ISSN 2525-1112|Año 3 No. 6 Julio-Diciembre 2018, pp. 209-220

alto, podríamos considerarlos como "estrategias de inserción internacional dependiente con márgenes de autonomía".

Este tipo de análisis multidisciplinar, pretende profundizar el análisis social, no solamente para mejorar nuestra comprensión del pasado, sino también para cuestionar, proponer y propiciar formas más autónomas de inserción de nuestro país en el sistema internacional.

\section{Bibliografía}

Aguirre Lanari, J. R. (1996). Una ruta transitable. En S. Jalabe (Comp.), La política exterior argentina y sus protagonistas, 1880-1995 (pp. 323-344). Buenos Aires: Grupo Editor Latinoamericano.

Cisneros, A. y Escudé, C. (1999). Historia general de las relaciones exteriores de la República Argentina. Buenos Aires: Grupo Editor Latinoamericano. Recuperado el 15 de julio de 2016, de http://www.argentina-rree.com/11/11-066.htm

Cujabante, X. (2009). La seguridad internacional: evolución de un concepto. Revista de Relaciones Internacionales, Estrategia y Seguridad, 4(2), 9319. https://doi.org/10.18359/ries.138

Escudé, C. (1983) .Gran Breta-a, Estados Unidos y la declinación argentina (19421949), Buenos Aires: Editorial De Belgrano.

Lanús, A. (1984). De Chapultepec al Beagle (1945-1980). Buenos Aires: Hyspamérica.

Míguez, M. C. (2011). La relación entre la política económica interna y la política exterior durante el proyecto desarrollista argentino (1958-1962). Contemporánea, 2(2), 53-78.

Míguez, M. C (2012). Illia y Santo Domingo: de las columnas de Primera Plana al golpe de estado. Ciclos en la Historia, la Economía y la Sociedad, XX(XXI), 39-40.

Míguez, M. C (2013). Los partidos políticos y la política exterior argentina Buenos Aires: Ariel.

Míguez, M. C. (2014). Apuntes histórico-conceptuales para la clasificación de la política exterior argentina de la posguerra hasta la actualidad. En M. Rapoport, B.

Míguez, M. C. (2015). El canciller argentino Miguel Ángel Zavala Ortiz y la revisión del principio de no intervención en la Argentina de la década de 1960. Historia del Derecho (50), 68-91.

Morgenfeld, L. (2013). Relaciones peligrosas. Argentina y Estados Unidos. Buenos Aires: Capital Intelectual.

Paradiso, J. (1993). Debates y trayectoria de la política exterior argentina. Buenos Aires: Grupo Editor Latinoamericano.

Perina, R. (1988). El estudio de la política exterior y de las relaciones internacionales argentinas. En R. Perina y R. Russell (Eds.), Argentina en el mundo (pp. 11-18), Buenos Aires: Grupo Editor Latinoamericano.

Potash, R. (1981). El ejército y la política en la Argentina 1945-1962. Buenos Aires: Sudamericana. 
Puig, J. C. (1980). Doctrinas internacionales y autonomía latinoamericana. Caracas: Instituto de Altos Estudios de América Latina, Universidad Simón Bolívar.

Puig, J. C. (1984). América Latina: políticas exteriores comparadas. Buenos Aires: Grupo Editor Latinoamericano.

Puig, J. C. (1988). Política internacional argentina. En R. Perina y R. Russell (Eds.), Argentina en el mundo (1973-1987) (pp. 19-45). Buenos Aires: Grupo Editor Latinoamericano.

Rapoport, M. (1981). Gran Breta-a, Estados Unidos y las clases dirigentes argentinas (1940-1945). Buenos Aires: Editorial De Belgrano.

Rapoport, M. (1997). El laberinto argentino. Buenos Aires: Eudeba.

Rapoport, M. y Spiguel, C. (2005). Política exterior argentina. Poder y conflictos internos (1880-2001). Buenos Aires: Capital Intelectual.

Rouquié, A. (1994). Poder militar y sociedad política en la Argentina, Tomo II, (19431973). Buenos Aires: Emecé.

Russell, R. (1988). Argentina y la política exterior del régimen autoritario (19761983): una evaluación preliminar. R. Perina y R. Russell, Roberto (Eds.), Argentina en el mundo (pp. 99-128). Buenos Aires: Grupo Editor Latinoamericano.

S. A. (5 de agosto de 1958). The Outlook for Argentina. National Intelligence Estimate, Foreign Relation of the United States, V, 495.

Simonoff, A. (2007). Los dilemas de la autonomía: la política exterior de Arturo Illia (1963-1966). Buenos Aires: Grupo Editor Latinoamericano.

Simonoff, A. (2015). Integración y autonomía en el pensamiento de Juan Carlos Puig. En J. Briceño Ruiz y A. Simonoff, A. (Comps.), Integración y cooperación regional en América Latina (pp. 121-142), Buenos Aires: Biblos. Vacs, A. (1984). Los socios discretos. Buenos Aires: Sudamericana.

Vázquez Ocampo, J. M. (1989). Política exterior argentina (1973-1983). De los intentos autonómicos a la dependencia. Buenos Aires: Centro Editor de América Latina. 